\title{
Evaluation of the optimal administrated activity in gated myocardial perfusion SPECT
}

\author{
Noha G. Madian¹, Islam Maamoun², HossamYasin ${ }^{3}$ and Reem H. El-Gebaly ${ }^{1}$ \\ ${ }^{1}$ Department of Biophysics, Faculty of Science, Cairo University, Giza, Egypt \\ ${ }^{2}$ Nuclear Cardiology Department, Intensive Care Unit, Faculty of Medicine, Cairo University Hospitals, Giza, Egypt \\ ${ }^{3}$ Nuclear Medicine Department, King Fahd Unit, Faculty of Medicine, Cairo University Hospitals, Giza, Egypt
}

\begin{abstract}
Prominence of radionuclide myocardial perfusion imaging (MPI) using single photon emission computed tomography (SPECT) has been investigated and shown to have high precision and predictive value. Improvement of the quality care for patients could be obtained by decreasing the radiation dose and increasing the image quality. The objective of this work is quantitative evaluation of the injected dose in MPI. 32 patients were categorized into 4 groups; each group received a different radiant dose. Image quality indices (Heart/Background (H/B), Heart/Lung (H/P) and Heart/ Liver $(\mathrm{H} / \mathrm{L}))$ ratios and cardiac ejection fraction $(\mathrm{EF} \%)$ were determined. It was observed from the images, that there is no substantial alteration in image quality among the groups. It was concluded that the most optimal dose providing the best image is the lowest one.
\end{abstract}

Key words: Myocardial perfusion imaging - Quantitative evaluation - Image quality - Image quality indices

\section{Introduction}

Myocardial perfusion imaging (MPI) in combination with single photon emission computed tomography (SPECT) is a crucial technique used in knowing the performance of the heart in patients which are susceptible to coronary artery disease (CAD) (Doukky et al. 2016).

The American Society of Nuclear Cardiology (ASNC) achieved a radiation effective dose (ED) of $9 \mathrm{mSv}$ in $50 \%$ by 2014 (equivalent to 3 years of US background radiation) for SPECT or photon emission computed tomography (PET) MPI patients (Cerqueira et al. 2010). It was found that the median radiation exposure for SPECT MPI to be $12.8 \mathrm{mSv}$ (range, 2-41 mSv) per study. The outcomes of the ASCN were achieved by $2.6 \%$ of SPECT laboratories. The SPECT findings from 2015 do not substantially differ from the previous report by Jerome et al. from 2012 to 2013. In the United States, there was no significant change in overall radiation exposure for SPECT MPI. Imaging for stress-only remains underutilized, and thallium remains present in a substantial

Correspondence to: Noha G. Madian, Biophysics Department, Faculty of Science, Cairo University, Giza, Egypt

E-mail: gamal_noha@yahoo.com amount of studies (8.4\%). In contrast, cardiac PET MPI utilization has increased since 2012 and has resulted in significantly reduced radiation exposure in 2015. This reduction is likely because of increased use of 3-dimensional (3D) imaging systems (Desiderio et al. 2018). These radiation estimates from the IAC database are supported by studies from the International Atomic Energy Agency (IAEA) Nuclear Cardiology Protocols Study (INCAPS) investigators who report similar estimates of SPECT radiation exposure, but with an increased prevalence of stress-only imaging in 50 selected nuclear laboratories in the United States. Few of these investigators indicate SPECT MPI with low dose (Desiderio et al. 2018).

For cardiovascular imaging, radiation protection was recently improved by the American Heart Association which recommended a process through which patients were treated. The process includes radiation risks, expected benefits and clinical justification. This recommendation was designed as class I (beneficial) with level of evidence $\mathrm{C}$ (expert consensus) indicating the lack of data in this field (Fazel et al. 2014).

Despite the low percentage of SPECT laboratories achieving ASNC goals, techniques are available to reduce radiation exposure and do not necessarily require purchase of new equipment. Reducing patient radiation exposure by protocol 
selection could easily be addressed by tracer choice (neither thallium nor dual-isotope), doses dependent on the weight and the use of functional testing as stress-only approaches. The IAC database demonstrates that even in 2015, 8.4\% of patient studies were using thallium in MPI protocols, despite ASNC recommendations against this practice. Data supporting the stress-first/stress-only concept demonstrate only $8 \%$ to $15 \%$ of all SPECT MPI studies are positive for ischemia, making stress-only imaging in a substantial percentage of patients feasible. Stress-only imaging has been recommended by professional societies for many years as a method of reducing radiation exposure by as much as 30 to $70 \%$, but in $2015,<0.5 \%$ of IAC laboratories used this practice (Desiderio et al. 2018).

In nuclear cardiology, it is important to reduce patient radiation exposure. Several trials were made in the last few years in order to increase photon sensitivity in nuclear cardiology scanner hardware, applying new software methods for SPECT reconstruction on conventional and enhance SPECT image quality with lower count statistics (Borges et al. 2007; DePuey et al. 2008, 2009, 2011, 2012; Vija et al. 2008; Maddahi et al. 2009; Venero et al. 2009; Druz et al. 2011; Duvall et al. 2018). Lately the SPECT regulations improve dose reduction by minimizing the use of Tl-201, adjusting the injected activity which is weight-dependent, using stress-only protocols, using new reconstruction algorithms and new cardiac camera technologies. All these parameters can reduce the radiation dose from a Tc99m-based study to $\leq 5 \mathrm{mSv}$ if they are used individually and to $2.6 \mathrm{mSv}$ or even less than $1 \mathrm{mSv}$ if they are used in combinations (Wells 2020). Correspondingly, SPECT regulations praised to exploit the accuracy of the test by decreasing imaging artifacts like attenuation effects. These artifacts can be reduced by using ECG-gated acquisitions and taking images in two different patient positions. This recommendation gives an indirect and unclear solution with more information without any increasing in radiation exposure to the patient (Wells 2020).

SPECT MPI applying novel scanners and software for stress-only with low-radiotracer-dose protocols (half-dose or less than half-dose), had a small radiation dose compared to standard rest-stress MPI protocols (Dorbala et al. 2015). It was established that the MPI images of reduced dose by $19 \%$ protocol were considered equivalent to full dose protocol with high image quality (Melby et al. 2012). Increasing pitch decreases dose by reducing the time needed to cover a given axial field-of-view and thus decreases the duration of radiation exposure. Increased pitch also spreads out the distance between adjacent acquired slices and may lead to decreased resolution (Wells 2020).

It was found that MPI half time imaging method produce high quality diagnostic image in comparison with full time imaging (Ali et al. 2009). Evolution for MPI cardiac reconstruction method, there is no significant difference in image quality between half dose and full dose images (Zafrir et al. 2012). This is true in case of obese patients by applying MPI half-dose reduction method. It was found that MPI procedure is feasible and can be performed in one day (Ali et al. 2009; Zafrir et al. 2012, 2013).

The diagnostic information increases by increasing the administered activity above a threshold point. The increase of the administered activity has no significance when an acceptable image quality has been reached. For each procedure, the administration activity increases to certain value known as optimum activity below which poor image quality and errors in diagnosis occur (Smith 1987). The indices of image quality (Heart/Background (H/B), Heart/Lung (H/P) and Heart/Liver $(\mathrm{H} / \mathrm{L})$ ratios) were used for evaluation of the image quality in nuclear cardiology (Ali et al. 2009). Automated 3D software is used for Left ventricle ejection fraction (LVEF), which need some geometrical change in LV cavity. The volume of LV cavity can be determined from this software in order to know the End diastolic volume (EDV) and the End systolic volume (ESV) (Zafrir et al. 2013).

The optimum injected dose of $99 \mathrm{mTc}$-MIBI for quantitative assessment of image quality in comparison to the standard injected dose was previously investigated (Melby et al. 2012). The purpose of the current study is to continue and to determine the optimum injected dose in SPECT MPI studies.

\section{Materials and Methods}

Patients were prepared in fasting state before the test ( 6 hours at least) and stopped cardiac and hypertension medications before the test for 48 hours. 32 patients complaining from chest have performed MPI studies (Melby et al. 2012). All the patients were grouped into four groups, each receiving different dose (370, 555, 740 and $925 \mathrm{MBq})$. Tc-99m Sestamibi eluted from MON-TEK Generator ${ }^{99 \mathrm{~m}} \mathrm{Mo} /{ }^{99 \mathrm{~m}} \mathrm{Tc}$ were administrated to each group with different values (El-Gebaly et al. 2014). Each group was injected intravenously and then scanned with dual head collimator LEHR gamma camera (Symbia one Siemens) (El-Gebaly et al. 2014).

The guidelines of the institution are required for all human experimental investigations.

\section{Administrated activity preparation}

MON-TEK Generator ${ }^{99 \mathrm{~m}} \mathrm{Mo} /{ }^{99} \mathrm{~m}$ Tc was used as a source for ${ }^{99 \mathrm{~m}} \mathrm{Tc}$. After this elution process, $9250 \mathrm{MBq}$ of ${ }^{99 \mathrm{~m}} \mathrm{TC}$ was added to MIBI vial and heated $10 \mathrm{~min}$ in water bath. The administrated activity required was removed from the labeled vial and measured by the dose calibrator to get the required activity. The waste of the administrated activity in the syringe after patient injection was measured by the dose calibrator. The real injected activity was calculated by 
subtraction the waste activity post injection from the total activity prepared before injection. Each group was administrated the injected dose of Tc-99m Sestamibi with stress study in nuclear cardiology and after $45 \mathrm{~min}$, patient is scanned at high resolution by dual head collimator LEHR gamma camera (Symbia Siemens SPECT).

\section{Image processing}

Data were stored in adequate computer Symbia (Germany), after $720 \mathrm{~s}$ time scan using matrix of $64 \times 64$. Nuclear medicine image expert assessed the image quality of each study. Filtered back projection method (FBP) reconstructs images in order of 4 and cut off 0.5 .

\section{Image quality indices}

Administered activity, matrix dimension, equipment, acquisition protocol, resting time after injection and acquisition time are all factors that affect the image quality. In the regions of interest (ROIs), the number of counts and the rate in the heart $(\mathrm{H})$, liver $(\mathrm{L})$, lungs $(\mathrm{P})$ and background region (B) between the liver and the left ventricle were measured. After achieving all parameters, the optimum activity was determined due to high image quality which is equivalent to the standard group.

\section{Left ventricle ejection fraction (LVEF)}

\section{LVEF by echocardiography}

Patients with left lateral recumbent perform quantitative echocardiographic studies. Apical four chamber view indicated LVEF images. Images were obtained from the apical two- and four-chamber views by knowing the abnormalities of the wall. The American Society of Echocardiography recommends that an irregular rhythm indicate five consecutive beats (El-Gebaly et al. 2014). The end-diastolic and end-systolic volume was accessed. LVEF was calculated as: $\mathrm{LVEF}=(($ end-diastolic volume - end-systolic volume $) \div$ end-diastolic volume) $\times 100$.

\section{LVEF by SPECT}

Data acquisition after 30 to 60 min administered dose of Tc-99m Sestamibi intravenously at stress study. SPECT data was acquired with high resolution dual-head gamma camera (Symbia one, Siemens). A total of 64 images were obtained with of $720 \mathrm{~s}$ total acquisition time.

Left ventricular epicardial and endocardial margins were performed by Cedars Sinai, computer-detection methods. Automated software was used to calculate the resting global LVEF from the gated SPECT images (El-Gebaly et al. 2015).

\section{Statistical analysis}

ANOVA and Post-Hock tests Statistical Package for the Social Sciences SPSS were used to compare between groups and to evaluate the significant values of the image quality indices.

\section{Results}

The image quality of the four groups showed no significant difference as was reported in a previous study (Smith 1987). The variation between image quality indices and administrated activity was evaluated (Tables 1 and 2).

Table 1. Relation between the image quality indices and the administrated activity

\begin{tabular}{|c|c|c|c|}
\hline Dose $(\mathrm{MBq})$ & $\mathrm{H} / \mathrm{B}$ & $\mathrm{H} / \mathrm{L}$ & $\mathrm{H} / \mathrm{P}$ \\
\hline \multirow[t]{8}{*}{370} & 2.1 & 1.3 & 2.6 \\
\hline & 2.9 & 2.2 & 3 \\
\hline & 1.2 & 1.01 & 1.9 \\
\hline & 2 & 0.7 & 1.7 \\
\hline & 2.6 & 1.1 & 2.6 \\
\hline & 2 & 1.3 & 2.4 \\
\hline & 2.1 & 1.4 & 2.3 \\
\hline & 2.2 & 0.9 & 2.3 \\
\hline \multirow[t]{8}{*}{555} & 2.9 & 1.07 & 2.2 \\
\hline & 2.5 & 1.02 & 2.5 \\
\hline & 2.4 & 1.4 & 2.6 \\
\hline & 2.4 & 0.7 & 2.1 \\
\hline & 2.6 & 1.4 & 2.5 \\
\hline & 3.6 & 1.2 & 2 \\
\hline & 2.4 & 0.8 & 2 \\
\hline & 2.5 & 1.8 & 2.2 \\
\hline \multirow[t]{8}{*}{740} & 2.7 & 0.9 & 2.6 \\
\hline & 2.4 & 1.2 & 2.7 \\
\hline & 2.1 & 1 & 2.3 \\
\hline & 2 & 1.1 & 2.2 \\
\hline & 2.1 & 1 & 2 \\
\hline & 2 & 1.1 & 2.2 \\
\hline & 2 & 1.02 & 2.2 \\
\hline & 2.7 & 1.8 & 2.5 \\
\hline \multirow[t]{8}{*}{925} & 2.5 & 0.9 & 2.2 \\
\hline & 2.7 & 1.07 & 2.5 \\
\hline & 2.5 & 1.9 & 2.3 \\
\hline & 2.1 & 1.9 & 2.4 \\
\hline & 2 & 1.2 & 2 \\
\hline & 2.1 & 0.9 & 2.3 \\
\hline & 1.6 & 0.8 & 1.6 \\
\hline & 2.1 & 1.0 & 1.8 \\
\hline
\end{tabular}

H/B, Heart/Background; H/P, Heart/Lung; H/L Heart/Liver (ratios of image quality). For more detail information, see section "Materials and Methods". 


\section{A}

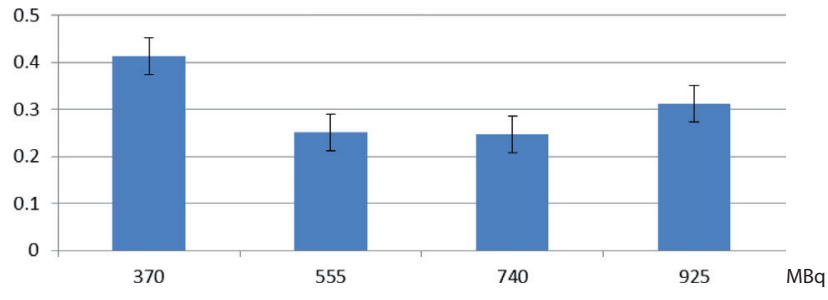

C

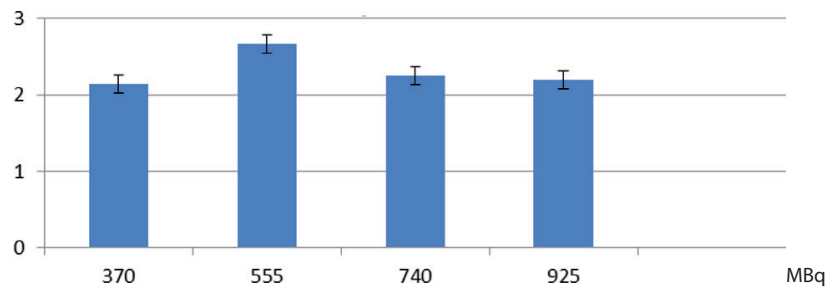

There is no significance difference between image quality indices $(\mathrm{H} / \mathrm{B}, \mathrm{H} / \mathrm{P}$ and $\mathrm{H} / \mathrm{L}$ ratios) for different groups $(p>0.05)$ as shown in Figure 1. Since the gated MPI is used for calculation of ejection fraction statistically, no significant difference was found between the ejection fraction calculated by SPECT at different administrated activities and the ejection fraction calculated by echocardiography where $p>0.05$ (Table 3) for each group in Figure 2. The mean value of the ejection fraction for all studies was $60 \pm 15 \%$.

\section{Discussion}

The target of the work is to study the relation between the diagnostic image quality and patient radiation protection. In 2014 , a total radiation exposure of $\leq 9 \mathrm{mSv}$ stated by ASNC should be achieved in 50\% of SPECT or PET MPI studies (Schiller et al. 1989). The diagnostic value increases with increasing administered activity. The results of image quality are independent on the administered activity. Optimum activity should be improved for each procedure below which poor quality images and possible errors occurs (Cerqueira et al. 2010).

Table 2. Image quality indices for the different injected activities

\begin{tabular}{lccc}
\hline $\begin{array}{c}\text { Dose } \\
(\mathrm{MBq})\end{array}$ & $\mathrm{H} / \mathrm{L}$ & $\mathrm{H} / \mathrm{B}$ & $\mathrm{H} / \mathrm{P}$ \\
\hline 370 & $2.3500 \pm 0.410$ & $1.23875 \pm 0.4137$ & $2.1375 \pm 0.452$ \\
555 & $2.2625 \pm 0.250$ & $1.084286 \pm 0.250$ & $2.6625 \pm 0.338156$ \\
740 & $2.3375 \pm 0.23867$ & $1.14000 \pm 0.247$ & $2.2500 \pm 0.28122$ \\
925 & $2.1375 \pm 0.35054$ & $1.20875 \pm 0.311$ & $2.2000 \pm 0.44341$ \\
\hline
\end{tabular}

Data are means \pm SD. For abbreviations see Table 1 .
B

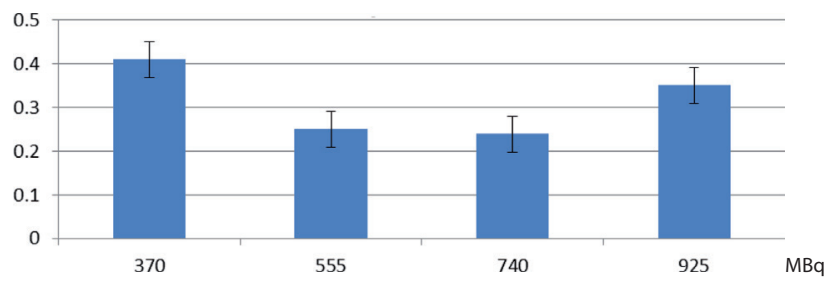

Figure 1. The relation between H/B (Heart/Background; A), H/L (Heart/Liver; B), H/P (Heart/Lung; C) and the administrated activity.

In the myocardial perfusion imaging SPECT, $1100 \mathrm{MBq}$ administrated activity, $11.4 \mathrm{mSv}$ effected dose and $70-\mathrm{kg}$ patients standard doses were taken. We can increase the dose by increasing the patient weight by $1.48 \mathrm{MBq} / \mathrm{kg}$ for 99mTc-MIBI (Holly et al. 2010; Cheng and Xiaohong 2019). Based on the protocol used and by increasing the acquisition time and changing the software, hardware and the standard doses, dosage can be reduced (Acampa et al. 2016; Cheng and Xiaohong 2019).

There are two points of view; one is the qualitative view, in which an expert proves that all image qualities are accepted. Second is the quantitative view, in which there is no significance difference in image quality indices between groups.

The ratios between the counts in the heart and background, lung and liver in different groups were not significantly different, because in stress study the heart injected dose is $1.5 \%$ while, the injected dose of other organs is $98.5 \%$ (ICRP 1998).

The indices of image quality are better instead of their higher values. It means that the number of counts in the heart is higher than in other organs considered as background (Diaz et al. 2003). The image quality indices shown in Figure 1 had no significant difference between the four groups in which $p$ value $>0.05$, so stress study in myocardial perfusion SPECT is feasible with $370 \mathrm{MBq}$.

As a result, it was observed that the dose delivered to the organs was increased by increasing the injected dose, while the opposite was true for a low injection dose. Rest and stress imaging undergo tissue attenuation artifacts due to anatomic structures leading to perfusion abnormalities mimicking ischemia. These abnormalities resulted in incorrect diagnosis of myocardial scar/infarction (Fleischmann et al. 1998; Picano et al. 2000; Beller 2019). 
The relation between the Echocardiography Ejection Fraction (EF\%) and SPECT EF\% was not significant for all groups, so the quantitative functions as EDV, end systolic volume (ES) and EF\% can be measured accurately with low administrated activity $(370 \mathrm{MBq})$. The optimum activity was $370 \mathrm{MBq}$ Tc-99m-MIBI for stress study in which optimum image quality was achieved and this agrees with ALARA (as low as possible reasonable achievable) concept.

This research supports following the recommendations of ASNC, as follows (Cerqueira et al. 2010):

(1) Use radionuclide with small $\mathrm{T}_{1 / 2}$ such as Tc-99 $\mathrm{m}$ and PET tracers.

Table 3. Represents the relation between the echocardiography ejection fraction and the ejection fraction of different groups calculated by myocardial perfusion SPECT

\begin{tabular}{|c|c|c|}
\hline Dose (MBq) & Echo (EF\%) & SPECT (EF\%) \\
\hline \multirow[t]{8}{*}{370} & 45 & 48 \\
\hline & 56 & 58 \\
\hline & 60 & 64 \\
\hline & 58 & 60 \\
\hline & 65 & 65 \\
\hline & 68 & 70 \\
\hline & 50 & 52 \\
\hline & 66 & 68 \\
\hline \multirow[t]{8}{*}{555} & 47 & 50 \\
\hline & 50 & 52 \\
\hline & 55 & 55 \\
\hline & 54 & 56 \\
\hline & 64 & 67 \\
\hline & 62 & 63 \\
\hline & 68 & 65 \\
\hline & 70 & 68 \\
\hline \multirow[t]{8}{*}{740} & 60 & 60 \\
\hline & 65 & 64 \\
\hline & 70 & 71 \\
\hline & 45 & 46 \\
\hline & 50 & 53 \\
\hline & 75 & 79 \\
\hline & 70 & 69 \\
\hline & 68 & 67 \\
\hline \multirow[t]{8}{*}{925} & 63 & 67 \\
\hline & 65 & 64 \\
\hline & 67 & 70 \\
\hline & 59 & 60 \\
\hline & 59 & 62 \\
\hline & 49 & 52 \\
\hline & 45 & 48 \\
\hline & 60 & 62 \\
\hline
\end{tabular}

A 80

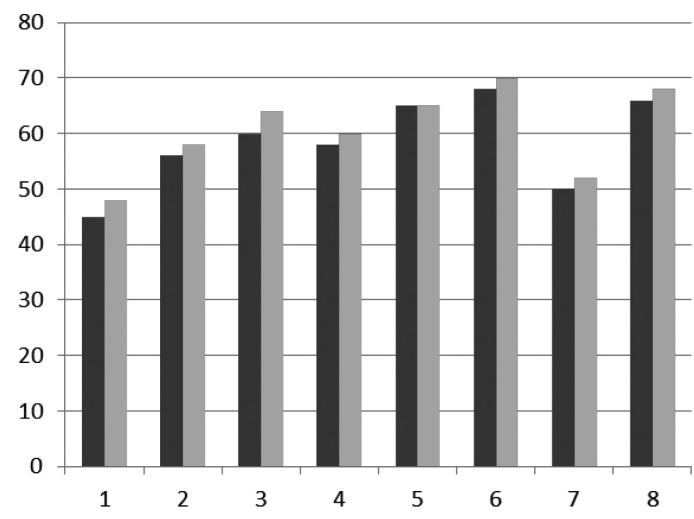

B

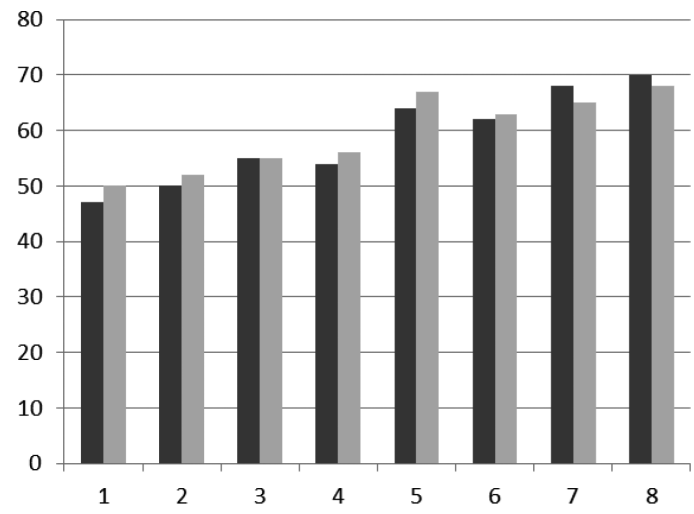

C

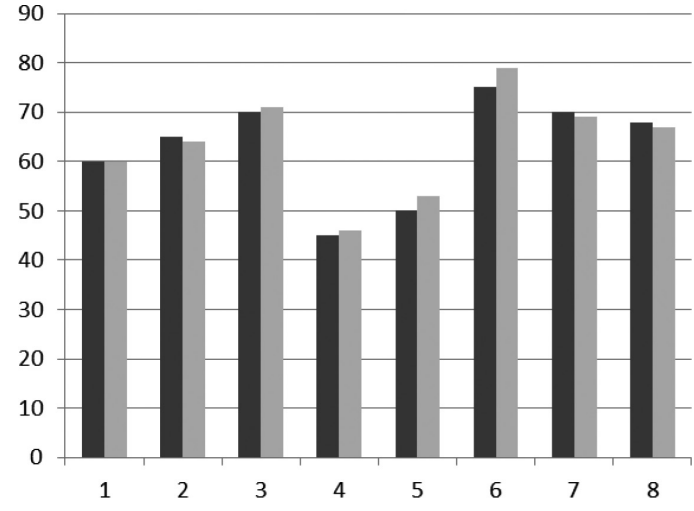

D 80

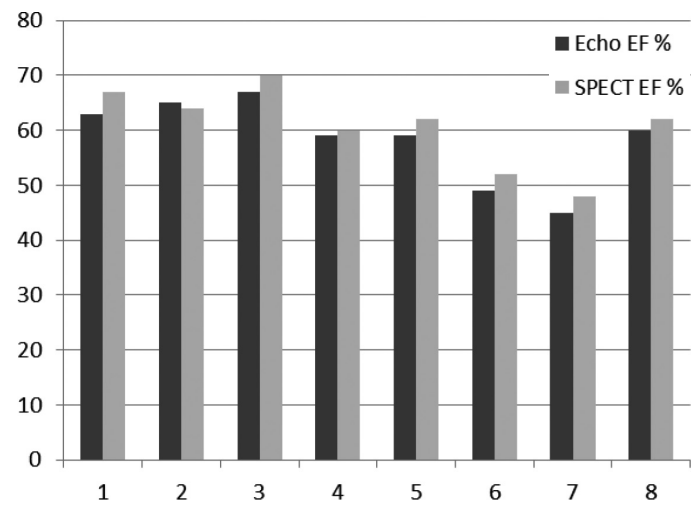

Figure 2. The relation between the echocardiography ejection fraction and the ejection fraction of $370 \mathrm{MBq}(\mathbf{A}), 555 \mathrm{MBq}(\mathbf{B}), 740 \mathrm{MBq}(\mathbf{C})$ and $925 \mathrm{MBq}(\mathrm{D})$ group calculated by myocardial perfusion SPECT. 
(2) Study the stress test only.

(3) Using dosage dependent on weight.

(4) Iterative reconstruction should be used for the processing of SPECT and PET MPI.

(5) Two acquisition detectors $\left(180^{\circ}\right)$ in Anger camera MPI studies.

(6) The geometry of SPECT imaging should be of high sensitivity.

(7) Using of high sensitivity collimators can reduce the injected radiation.

(8) Widening the energy window requires further validation prior to clinical practice.

(9) Continuous acquisition, and count consistency methods require further validation before implementation into clinical practice.

\section{Conclusion}

$370 \mathrm{MBq}$ administrated dose gives the optimum results in image quality in comparison with high dose $925 \mathrm{MBq}$. This is in agreement with ALARA.

\section{References}

Acampa W, Buechel RR, Gimelli A (2016): Low dose in nuclear cardiology: state of the art in the era of new cadmiumzinc-telluride cameras. Eur. Heart J. Cardiovasc. Imaging 17, 591-595

https://doi.org/10.1093/ehjci/jew036

Ali I, Ruddy TD, Almgrahi A, Anstett FG, Wells RG (2009): Halftime SPECT myocardial perfusion imaging with attenuation correction. J. Nucl. Cardiol. 50, 554-562 https://doi.org/10.2967/jnumed.108.058362

Beller AG, Zaret BL (2019): Contributions of nuclear cardiology to diagnosis and prognosis of patients with coronary artery disease. Circulation 101, 1465-1478 https://doi.org/10.1161/01.CIR.101.12.1465

Borges-Neto S, Pagnanelli RA, Shaw LK, Honeycutt E, Shwartz SC, Adams GL, Coleman RE (2007): Clinical results of a novel wide beam reconstruction method for shortening scan time of Tc-99m cardiac SPECT perfusion studies. J. Nucl. Cardiol. 14, 555-565 https://doi.org/10.1016/j.nuclcard.2007.04.022

Cerqueira MD, Allman KC, Ficaro EP, Hansen CL, Nichols KJ, Thompson RC, Van Decker WA, Yakovlevitch M (2010): Recommendations for reducing radiation exposure in myocardial perfusion imaging. J. Nucl. Cardiol. 17, 709-718 https://doi.org/10.1007/s12350-010-9244-0

Cheng J, Xiaohong O (2019): Semi-quantitative analysis of 99mTcMIBI SPECT/CT in distinguishing between sHPT and tHPT. J. Nucl. Med. 60, 1092

DePuey EG, Gadiraju R, Clark J, Thompson L, Anstett F, Shwartz SC (2008): Ordered subset expectation maximization and wide beam reconstruction "half-time“ gated myocardial perfusion
SPECT functional imaging: a comparison to „full-time“ filtered back projection. J. Nucl. Cardiol. 15, 547-563

https://doi.org/10.1016/j.nuclcard.2008.02.035

DePuey EG, Bommireddipalli S, Clark J, Thompson L, Srour Y (2009): Wide beam reconstruction "quarter-time“" gated myocardial perfusion SPECT functional im-aging: a comparison to "full-time“ ordered subset expectation maximum. J. Nucl. Cardiol. 16, 736-752 https://doi.org/10.1007/s12350-009-9108-7

DePuey EG, Bommireddipalli S, Clark J, Leykekhman A, Thompson LB, Friedman M. (2011): A comparison of the image quality of full-time myocardial perfusion SPECT vs wide beam reconstruction half-time and half-dose SPECT. J. Nucl. Cardiol. $18,273-280$ https://doi.org/10.1007/s12350-011-9340-9

DePuey EG, Ata P, Wray R, Friedman M (2012): Very low-activity stress/high-activity rest, single-day myocardial perfusion SPECT with a conventional sodium iodide camera and wide beam reconstruction processing. J. Nucl. Cardiol. 19, 931-944 https://doi.org/10.1007/s12350-012-9596-8

Desiderio MC, Lundbye JB, Baker WL, Farrell MB, Jerome SD, Heller GV (2018): Current status of patient radiation exposure of cardiac positron emission tomography and single-photon emission computed tomographic myocardial perfusion imaging. Circ. Cardiovasc. Imaging 11, e007565 https://doi.org/10.1161/CIRCIMAGING.118.007565

Díaz MP, Aparicio EE, Rizo OD, Díaz RR, Rodríguez CH. (2003): Administered activity optimization in 99mTc-MAG3 renography for adults. J. Nucl. Med. Technol. 31, 216-221

Dorbala S, Blankstein R, Skali H, Park MA, Fantony J, Mauceri C, Semer J, Moore SC, Di Carli MF (2015): Approaches to reducing radiation dose from radionuclide myocardial perfusion imaging. J. Nucl. Med. 56, 592-599 https://doi.org/10.2967/jnumed.112.115097

Doukky R, Frogge N, Appis A, Hayes K, Khoudary G, Fogg L, Williams KA Sr. (2016): Impact of appropriate use on the estimated radiation risk to men and women undergoing radionuclide myocardial perfusion imaging. J. Nucl. Med. 57, 1251-1257 https://doi.org/10.2967/jnumed.115.167205

Druz RS, Phillips LM, Chugkowski M, Boutis L, Rutkin B, Katz S (2011): Wide-beam reconstruction half-time SPECT improves diagnostic certainty and preserves normalcy and accuracy: a quantitative perfusion analysis. J. Nucl. Cardiol. 18, $52-61$ https://doi.org/10.1007/s12350-010-9304-5

Duvall WL, Tandon TS, Henzlova MJJ (2018): The time is now: Dose reduction for myocardial perfusion imaging. J. Nucl. Cardiol. 25, 131-133 https://doi.org/10.1007/s12350-016-0639-4

El-Gebaly RH, Maamoun IK, Madian NG (2014): Quantitative evaluation of the administrated dose affecting image quality in myocardial perfusion SPECT. J. XRay Sci. Technol. 22, 529-537 https://doi.org/10.3233/XST-140443

El-Gebaly RH, Rageh MM, Adel M (2015): Evaluation of varying physical acquisition parameters in gamma camera gated cardiac SPECT. J. XRay Sci. Technol. 23, 453-461 https://doi.org/10.3233/XST-150500 
Fazel R, Gerber TC, Balter S, Brenner DJ, Carr JJ, Cerqueira MD, Chen J, Einstein AJ, Krumholz HM, Mahesh M, et al.(2014): Approaches to enhancing radiation safety in cardiovascular imaging": a scientific statement from the American Heart Association. Circulation 130, 1730-1748 https://doi.org/10.1161/CIR.0000000000000048

Fleischmann KE, Hunink MG, Kuntz KM, Douglas PS (1998): Exercise echocardiography or exercise SPECT imaging? A meta-analysis of diagnostic test performance. JAMA 280, 913-920 https://doi.org/10.1001/jama.280.10.913

Holly TA, Abbott BG, Al-Mallah M, Calnon DA, Cohen MC, DiFilippo FP, Ficaro EP, Freeman MR, Hendel RC, Jain D, et al. (2010): Single photon-emission computed tomography. ASNC imaging guidelines for nuclear cardiology procedures. J. Nucl. Cardiol. 17, 941-973 https://doi.org/10.1007/s12350-010-9246-y

Smith H (1987): Protection of the patient in nuclear medicine. Ann. ICRP 4, 1-37 https://doi.org/10.1016/0146-6453(87)90007-8

ICRP (1998): Radiation dose to patients from radiopharmaceuticals (addendum to ICRP publication 53). Ann. ICRP 28, 1-126 https://doi.org/10.1016/S0146-6453(99)00006-8

Maddahi J, Mendez R, Mahmarian JJ, Thomas G, Babla H, Bai C, Arram S, Maffetone P, Conwell R (2009): Prospective multicenter evaluation of rapid, gated SPECT myocardial perfusion upright imaging. J. Nucl. Cardiol. 16, 351-357 https://doi.org/10.1007/s12350-009-9063-3

Melby P, Manson L, Johnson C, Kessler C (2012): The effect of Tc $99 \mathrm{~m}$ Sestamibi dose reduction on image quality and patient radiation exposure. J. Nucl. Med. 53, 2722

Picano E, Bedetti G, Varga A, Cseh E (2000): The comparable diagnostic accuracies of dobutamine-stress and dipyridamolestress echocardiographies: a meta-analysis. Coron. Artery Dis. 11, 151-159 https://doi.org/10.1097/00019501-200003000-00010
Schiller NB, Shah PM, Crawford M, DeMaria A, Devereux R, Feigenbaum H, Gutgesell H, Reichek N, Sahn D, Schnittger I, et al. (1989): Recommendations for quantitation of the left ventricle by two-dimensional echocardiography. American Society of Echocardiography Committee on Standards, Subcommittee on Quantitation of Two-Dimensional Echocardiograms. J. Am. Soc. Echocardiogr. 2, 358-367 https://doi.org/10.1016/S0894-7317(89)80014-8

Venero CV, Heller GV, Bateman TM, McGhie AI, Ahlberg AW, Katten D, Courter SA, Golub RJ, Case JA, Cullom SJ (2009): A multicenter evaluation of a new post-processing method with depth-dependent collimator resolution applied to full-time and half-time acquisitions without and with simultaneously acquired attenuation correction. J. Nucl. Cardiol. 16, 714-725 https://doi.org/10.1007/s12350-009-9106-9

Vija H, Chapman J, Ray M (2008): IQ•SPECT technology White Paper. Siemens Medical Solutions USA Molecular Imaging 2008, 1-7

Wells RG (2020): Dose reduction is good but it is image quality that matters. J. Nucl. Cardiol. 27, 238-240 https://doi.org/10.1007/s12350-018-1378-5

Zafrir N, Bental T, Solodky A, Ben-Shlomo A, Mats I, Hassid Y, Belzer D, Battler A, Gutstein A (2012): Feasibility of myocardial perfusion imaging with half the radiation dose in obese patients using ordered-subset expectation maximization with resolution recovery software. J. Nucl. Cardiol. 20, 111-119 https://doi.org/10.1007/s12350-012-9650-6

Zafrir N, Solodky A, Ben-Shlomo A, Mats I, Nevzorov R, Battler A, Gutstein A (2013): Feasibility of myocardial perfusion imaging with half the radiation dose in obese patients using orderedsubset expectation maximization with resolution recovery software. J. Nucl. Cardiol. 19, 704-712 https://doi.org/10.1007/s12350-012-9650-6

Received: March 17, 2020

Final version accepted: August 26, 2020 